\title{
Conocimientos y actitudes sobre anticoncepción de emergencia de los médicos de Atención Primaria en el área de salud de Toledo
}

\author{
F. López de Castro, G. Alejandre Lázaro*, J. López Díaz*, n. Campos Campos*, N. \\ Marañón HeInRICH*, S. Redondo de PedRo* \\ Especialista en Medicina de Familia y Comunitaria. Coordinador de la Unidad Docente \\ de Medicina de Familia y Comunitaria. *Residente de tercer año de Medicina de \\ Familia y Comunitaria. Unidad Docente de Medicina de Familia y Comunitaria. Toledo
}

\section{RESUMEN}

Fundamento: la anticoncepción de emergencia (AE) es una demanda creciente en Atención Prima ria $(A P)$.

Objetivo: valorar los conocimientos y actitudes sobre AE de los médicos de AP.

Diseño: descriptivo transversal, mediante en cuesta autocumplimentada.

Material y métodos: la población del estudio son los 336 médicos de AP del área. La encuesta incluye items sobre métodos conocidos, efectos se cundarios, contraindicaciones, demanda y actitud ante la misma, pauta empleada por los prescripto res, uso de antieméticos profilácticos, test de gestación previo y derivación al Centro de Orientación Familiar (COF).

Resultados: respondieron 139 (41,3\%). Un 8,0\% no conocía ningún método de AE; el 84,9\% citaba preparados hormonales, un 12,9\% DIU y un $3,5 \%$ otros. El efecto secundario considerado más frecuente fue vómitos/náuseas (69,7\%); el más grave, tromboembolismo $(35,9 \%)$ y la principal con traindicación, embarazo (84,8\%). El 93,5\% tuvie ron alguna demanda de $A E$ en el último año. Lo consideran abortivo el 27,5\%. Prescriben AE el $68,8 \%$. Este porcentaje es menor en aquéllos que lo consideran aborto. De los prescriptores, reali zan test de embarazo el 74,4\%. La pauta más utili zada $(54,1 \%)$ es la clásica de Yuzpe. Prescriben antieméticos profilácticos siempre el 30,6\%. Remi ten al COF siempre el 33,7\%. Si la solicitante fue ra menor, el 49,6\% de los encuestados recomenda. rían que volviera con un familiar mayor de edad y el 17,6\% prescribirían $A E$.
Knowledge and attitudes about emergency contra ception of Primary Health Care doctors in Toledo

\section{ABSTRACT}

Background: Emergency Contraception (EC) is an increasing demand in Primary Health Care (PHC).

Objective: to study the knowledge and attitudes about EC of doctors in PHC.

Design: cross sectional descriptive study th rough autofilled questionnaire.

Subjects and methods: the participans of our study were 336 doctors who worked in PHC. The question naire included items about known methods, side ef fects, contraindications, request and attitude about the same, regimen used by the prescriptors, used of prophilactic anti-nausea drugs, previous pregnancy test and referral to Family Planning Centre (FPC).

Results: it were responded 139 questionnaires (41.3\%). 8.0\% not known any EC methods; $84.9 \%$ named hormonal regimen, $12.9 \%$ Intrauterine Contraceptive Device (IUCD) and others a $3.5 \%$. It was considered as the most frecuent side effect vomiting/nausea (69.7\%); the most severe was ve nous thrombosis $(35.9 \%)$ and the main contraindi cation was pregnancy (84.8\%). The $93.5 \%$ had some request of EC during the last year. The met hod was considered as abortion in $27.5 \%$. EC was prescripted in $68.8 \%$. This percentage is less in those who considered it as an abortion. Prescrip tors made a pregnancy test in $74.4 \%$. The most used regimen was the Yuzpe one (54.1\%). It was al ways prescribed prophylactical antiemetic drugs in $30.6 \%$. They always refered to FPC in $33.7 \%$. If the demandant were less than 18 years old, the $49.6 \%$ of the requested recommended to come back with a grown-up and they prescribed EC in $17.6 \%$. 
Conclusiones: existen lagunas de conocimiento sobre la AE y una actuación heterogénea entre nuestros profesionales. Creemos necesario mejorar su formación y construir guías de actuación claras para atender correctamente esta demanda, cre ciente e importante.

Palabras clave: Anticoncepción postcoital. Co nocimientos. Actitudes y práctica. Atención Primaria de salud.
Conclusions: it were founded a lack of knowledge about EC and an heterogeneal actuation bet ween our professionals. We think that it is neces sary an improvement in their formation and make clear actuations guides to assist properly this im portant and increasing demand.

Key words: Postcoital contraception. Knowledge. Attitudes and practice. Primary Health Care.

\section{INTRODUCCIÓN}

Se denomina anticoncepción postcoital o de emergencia (AE) al conjunto de métodos empleados después de un coito sin protección que suponga riesgo de embarazo por haber sucedido en la fase fértil de la mujer, con el fin de evitar embarazos no deseados y dentro de un período máximo de tiempo $^{1}$. En nuestro país, en 1996, el 12,3\% de los embarazos acabaron en una interrupción voluntaria del mismo; este porcentaje llegó al 39,5\% en las mujeres menores de 20 años $^{2}$. Los embarazos no deseados son causa de aproximadamente 80.000 muertes en el mundo por abortos realizados en condiciones higiénicas deficientes ${ }^{3}$. Gran parte de estas muertes podrían ser evitadas con una adecuada educación sexual y más fácil acceso a métodos de planificación familiar, dentro de los cuales se encuentra la $\mathrm{AE}^{4}$.

Los métodos de $\mathrm{AE}$ son básicamente dos: preparados hormonales o implantación de un dispositivo intrauterino (DIU). Dentro de los hormonales, pueden usarse estrógenos solos o con progestágenos, progestágenos solos, danazol o mifepristona (RU486) ${ }^{5,6}$. El método más conocido, sin duda, es el de Yuzpe $^{7}: 200 \mu \mathrm{g}$ etinilestradiol (EE) + 1 mg levonorgestrel (LNG), administrados en 2 dosis separadas por 12 horas. En fechas muy recientes ha sido comercializado en nuestro país un preparado específico para $\mathrm{AE}$ con $0,75 \mathrm{mg}$ de levonorgestrel, sin estrógenos. Todos deben emplearse y actuar antes de la implantación del óvulo, que se acepta tiene lugar entre el $5^{\circ}$ y el $7^{\circ}$ día $^{8}$, siendo por lo general más eficaces cuanto más precoz es el tratamiento $0^{9-11}$. Habitualmente se recomienda no sobrepasar las 72 horas en el caso de AE hormonal ${ }^{12,13}$ o las 120 cuando se trata de DIU o mifepristona ${ }^{14,15}$, aunque se pueden ampliar estos plazos, advirtiendo a la mujer de su menor efectividad ${ }^{16}$.

La eficacia de la AE en la prevención de embarazos no deseados difiere según el método, aunque puede decirse que oscila entre el $74 \%$ para el de Yuzpe y el 99\% para el DIU ${ }^{17,18}$.

La AE se ha convertido en los últimos años en un tema de actualidad, apareciendo con relativa frecuencia en los medios de comunicación ${ }^{19,20} \mathrm{y}$ convirtiéndose en foco de investigación a nivel mundia ${ }^{21}$. Este fenómeno se ha debido al aumento de su utilización, sobre todo por los jóvenes y adolescentes ${ }^{22}$. Una parte importante de esta demanda asistencial recae en los profesionales de Atención Primaria; en gran medida a través de los servicios de atención continuada.

Este "nuevo" motivo de consulta se encuentra con una falta de homogeneidad en la actuación de los propios médicos ${ }^{23}$, posiblemente debida al desconocimiento de los métodos y sus pautas, corta experiencia en su uso, problemas éticos o temor a conflictos legales ${ }^{24,25}$; por todo lo anterior nos pareció relevante obtener información sobre la situación en nuestra área de salud, motivo por el cual diseñamos el presente estudio, cuyo objetivo fue valorar el grado de conocimiento, la actitud y el comportamiento sobre la $\mathrm{AE}$ de los médicos de Atención Primaria del área de salud de Toledo.

\section{MATERIAL Y MÉTODOS}

Se trata de un estudio descriptivo transversal, realizado a partir de los datos obtenidos de una encuesta autocumplimentada y anónima, distribuida a todos los médicos de Atención Primaria del área de Toledo, incluyendo médicos de refuerzo y residentes de tercer año de la especialidad de Medicina Familiar y Comunitaria, que también hacen guardias en los centros de salud.

Las encuestas se distribuyeron por los 32 centros de salud del área, entre noviembre de 2000 y enero de 2001; siendo remitidas una vez contestadas a la Unidad Docente. En todo el proceso se mantuvo el anonimato y la confidencialidad de los datos individuales.

La encuesta incluía preguntas sobre: conocimiento de algún método de $\mathrm{AE}$; efecto secundario más frecuente, más grave y contraindicaciones de AE hormonal; si lo consideraba abortivo; número de demandas en el último año (categorizado en $<5$, 5-10, >10); actitud ante la petición de AE (dando como posibles opciones: prescribir, derivar la de- 
manda, no dar respuesta u otras) y si su actitud variaba cuando se trataba de una menor de edad. En caso de derivación, se le solicitaba que especificara si era por "entender que debía ser atendida en el nivel especializado" o por "objeción de conciencia". A los que prescribían se les pidió la pauta habitualmente empleada [se consideraron pautas "dudosas" aquéllas que no se ceñían a las dosis y duración habituales: $100 \mu \mathrm{g}$ de $\mathrm{EE}+0,5 \mathrm{mg}$ de LNG / 12 horas (2 dosis) o 400-600 mg de danazol / 12 horas (2 dosis) $]^{6,26}$, si derivaban al Centro de Orientación Familiar (COF) una vez atendida, si utilizaban antieméticos profilácticos y si realizaban previamente test de gestación. Otras variables recogidas fueron: edad del médico, sexo, situación (refuerzo, titular o residente), medio laboral (rural, si es un municipio menor de 10.000 habitantes, o urbano) y realización o no de guardias.

Antes de iniciar el estudio se realizó un pilotaje con 25 médicos para detectar la presencia de errores en la encuesta, modificándose algunos ítems de la misma.

\section{RESULTADOS}

Se entregaron 336 cuestionarios, siendo contestados 139 (41,3\%). La proporción de respuestas entre los refuerzos y los titulares fue muy similar (39,0 y $39,1 \%$, respectivamente); mientras que los 11 residentes de Medicina de Familia contestaron todos.

En cuanto a las características de la muestra, la media de edad fue de 41,2 años (DE 8,6). El 45,3\% de los médicos eran mujeres. Los médicos titulares representan el $62,6 \%$, los refuerzos el $29,5 \%$ y los residentes el 7,9\%. Hubo 134 respuestas acerca del medio en que trabajaban: rural $70,1 \%$ y urbano $29,8 \%$. Hacían guardias el 70,8\%.

Respecto al conocimiento sobre métodos de AE, un $8,0 \%$ contestaba no conocer ninguno; el 84,9\% citaba los preparados estro/progestogénicos, un $12,9 \%$ mencionaba el DIU, un $2,8 \%$ la mifepristona y un $0,7 \%$ el danazol.

En las figuras 1 y 2 aparecen los datos sobre el efecto secundario considerado más frecuente (vómitos/náuseas, 69,7\%), el más grave (tromboembolismo, 35,9\%) y las contraindicaciones de la AE (embarazo, 84,8\%).

Contestaron que sí se les había planteado la demanda de $\mathrm{AE}$ en el último año el 93,5\%; de éstos, el $65,9 \%$ tuvieron menos de cinco casos, el 20,9\% entre cinco y diez, y el 13,2\% más de 10.

Consideraban que la $\mathrm{AE}$ es un método abortivo el $27,5 \%$. Esta opinión es más frecuente en hombres (28,0 vs 26,2\%), médicos de refuerzo (36,6 vs $22,3 \%$ en médicos titulares y $27,3 \%$ en residentes) y menores de 41 años $(32,1$ vs 22,6\%).
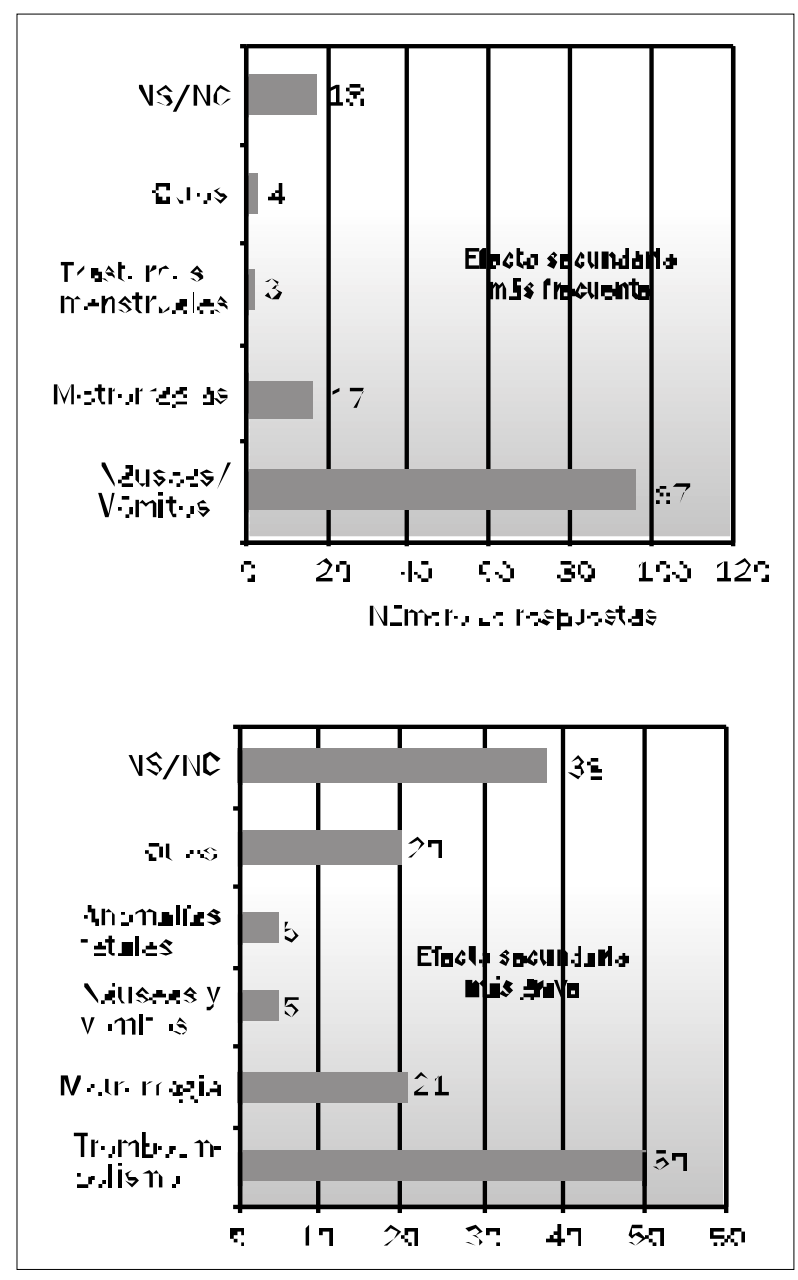

Figura 1

Efectos secundarios considerados más frecuentes y más graves.

En cuanto a la actitud (Fig. 3), afirmaron prescribir la AE el 68,8\%. Este porcentaje es mayor en mujeres, en aquéllos que trabajan en medio rural y en menores de 41 años. Los médicos de refuerzo son los menos prescriptores. También prescriben menos aquéllos que lo consideran aborto (Fig. 4).

De los prescriptores, dijeron realizar test previo de embarazo el 74,4\%. La pauta más utilizada por éstos es la clásica de Yuzpe de un día $(54,1 \%)$; la prolongan durante dos o tres días el $29,8 \%$ y emplean pautas más dudosas (por la duración o la dosis) el 16,1\%. Manifestaron remitir al COF siempre, tras resolver la demanda, el 33,7\%; a veces, el $54,7 \%$, y nunca el $11,6 \%$. Suelen prescribir antieméticos siempre el $30,6 \%$, algunas veces el $40,0 \%$ y nunca el 29,4\%.

En el caso de que la solicitante fuera menor de edad, el 49,6\% de los encuestados recomendarían que volviera con un familiar mayor de edad y el 17,6\% prescribirían AE (Fig. 3). La actitud con las menores de los que prescriben habitualmente se refleja en la figura 5 . 


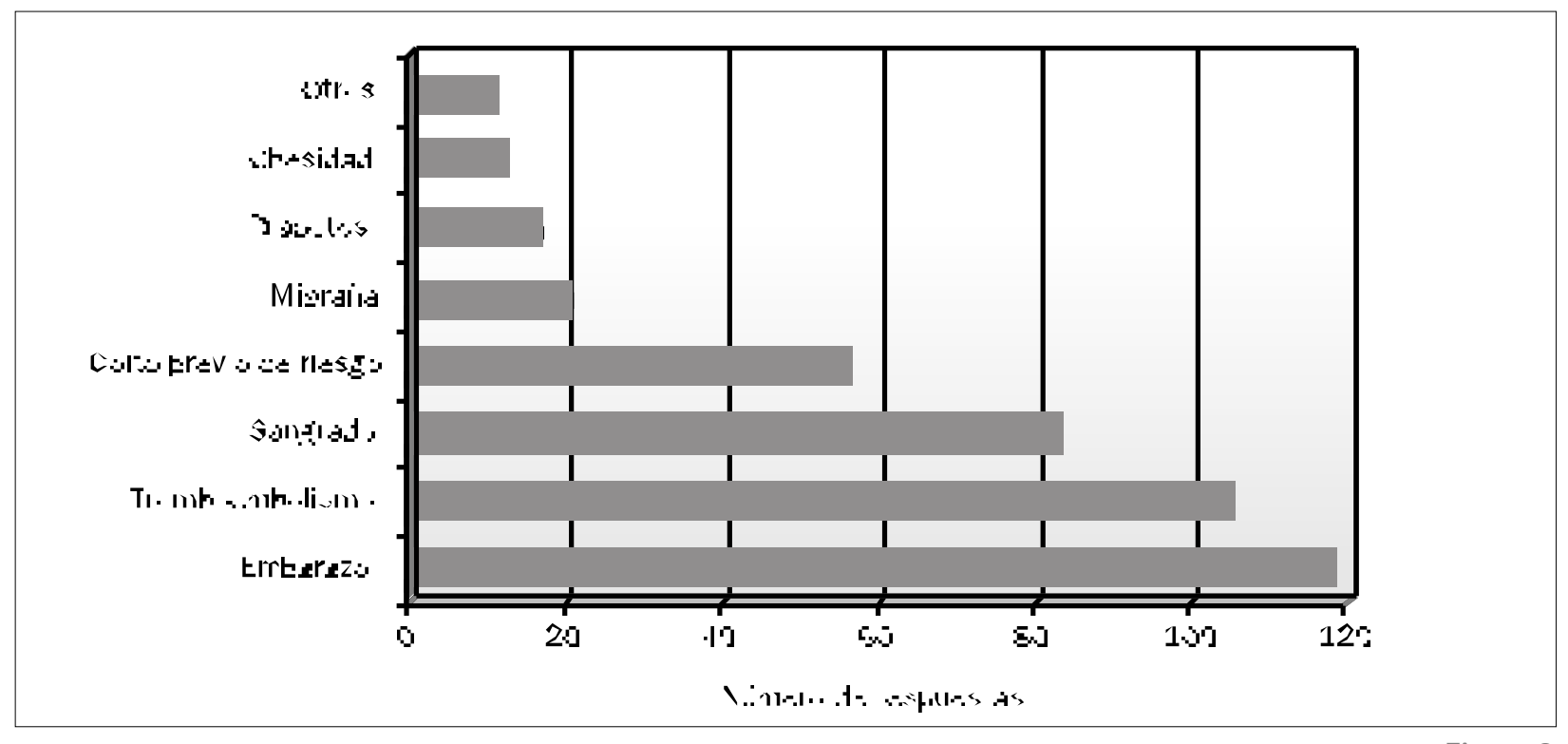

Figura 2

Contraindicaciones de la anticoncepción de emergencia.

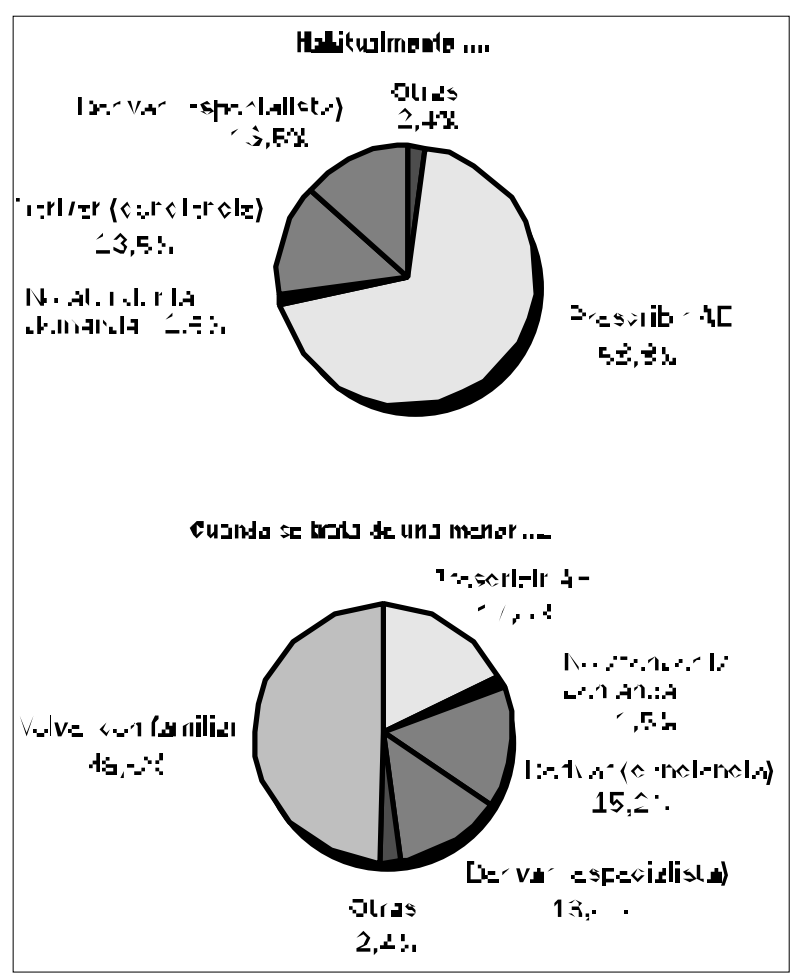

Figura 3

Actitud de los encuestados ante la demanda de anticoncepción de emergencia $(\mathrm{AE})$.

\section{DISCUSIÓN}

Antes de analizar los resultados, hemos de advertir que la baja tasa de respuesta de nuestro estudio (ligeramente superior al $41 \%$ ) podría suponer un sesgo. Al tratarse de una encuesta anónima, no podemos conocer las características de los no respondedores, pero la edad media y la distribución por sexos de la muestra son similares a la de la población de médicos del área (35\% mujeres, edad media 42,5 años). También la proporción de respondedores es similar entre médicos de equipo $(39,0 \%)$ y de refuerzo $(39,1 \%)$; mientras que en residentes fue del $100 \%$. Por ambas razones, consideramos que la muestra es suficientemente representativa, aunque con una mínima sobrepresencia de residentes que, por su reducido número, poco puede sesgar los resultados.

A la vista de los datos obtenidos, podemos concluir que existe cierta deficiencia en los conocimientos sobre $\mathrm{AE}$ en los médicos de nuestra área. De hecho, un $8 \%$ de los encuestados admiten no conocer ningún método, cifra más elevada que la de otros estudios ${ }^{27,28}$. Muy pocos mencionan otros métodos de AE distintos a la pauta de Yuzpe, como el DIU (el gran olvidado de la anticoncepción ${ }^{29,30}$, a pesar de que su tasa de fallos es menor del $1 \%{ }^{31}$ ), el danazol o la mifepristona, aunque en este último caso es comprensible, ya que su utilización como anticonceptivo de emergencia es relativamente reciente y sólo se emplea en algunos países ${ }^{32}$. Nadie menciona tampoco la pauta de AE con levonorgestrel solo, comercializada en España algunas semanas después de finalizar el estudio. En cualquier caso, el conocimiento de otros métodos de AE es inferior al observado por Carlón en Asturias ${ }^{28}$.

También comprobamos que se imputan a la pauta de Yuzpe las mismas contraindicaciones de la anticoncepción hormonal clásica (dosis más bajas pero mucho más prolongadas), cuando no existe ninguna evidencia de ello ${ }^{33,34}$. La ficha técnica del 


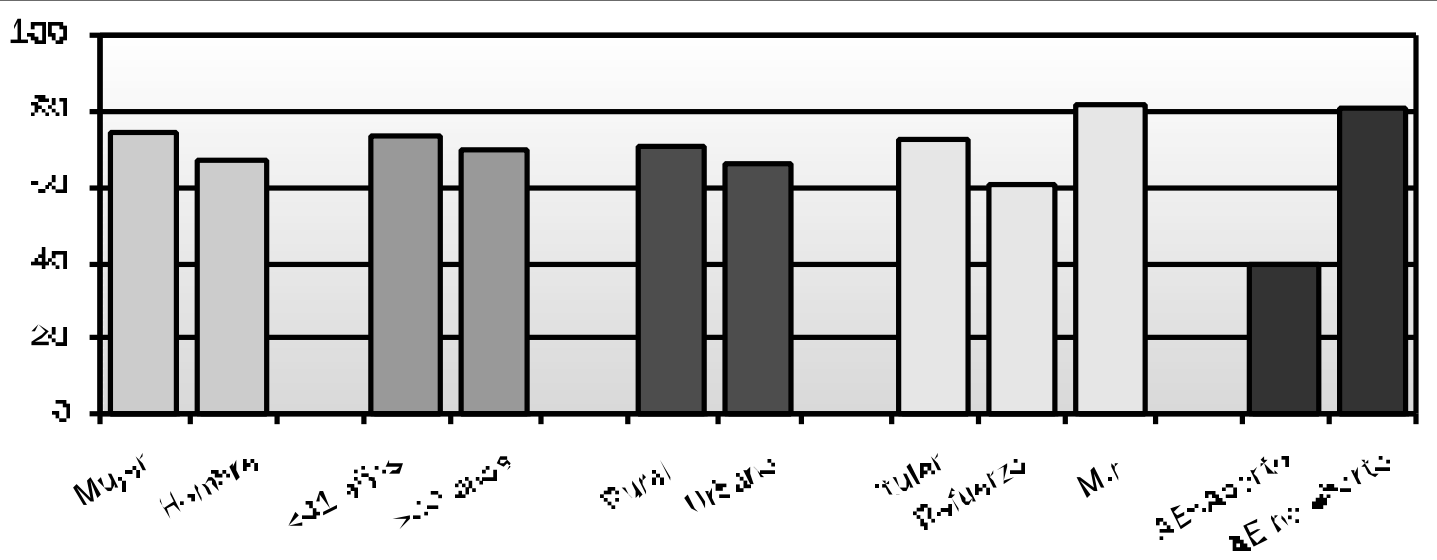

Figura 4

Porcentaje de prescriptores habituales de AE según varias características del médico

Preven ${ }^{\circledR}$, (un preparado específico para AE hormonal, no comercializado en nuestro país, a base de etinilestradiol y levonorgestrel) ${ }^{12}$ incluye como contraindicaciones: embarazo, tromboembolismo pulmonar, cardiopatía isquémica, hipertensión arterial severa, diabetes con complicaciones vasculares, migraña con focalidad neurológica, cáncer de mama, etc. (las habituales de los anticonceptivos orales), pero ni la OMS ni el Consorcio para la Contracepción de Emergencia han establecido ninguna contraindicación al uso de esta pauta, salvo el embarazo ${ }^{1,4}$. En la bibliografía consultada, algunos autores incluyen también la historia de migraña con aura como contraindicación absoluta o relati$\mathrm{va}^{14,35}$. Un estudio reciente de Vasilakis no encuentra mayor riesgo de tromboembolismo por el uso de AE hormonal ${ }^{36}$. Tampoco en Inglaterra, con una larga experiencia en $\mathrm{AE}$, se ha constatado una mayor incidencia de tromboembolismo en estas usuarias $^{37}$.

Donde sí parece existir un mayor grado de conocimientos es en lo referente a los efectos secundarios más frecuentes, ya que, efectivamente las náuseas y los vómitos aparecen muy a menudo $(50 \mathrm{y}$ $20 \%$, respectivamente) con la pauta de Yuzpe y la mitad con levonorgestrel o mifepristona ${ }^{38-41}$.

Un hecho preocupante es el elevado número de pautas "dudosas" que parecen prescribirse, ya que, aunque esta práctica es menor que la observada en otros trabajos ${ }^{28}$, puede suponer un incremento de los efectos secundarios si se da en exceso, o un descenso de la efectividad con mayor riesgo de embarazo, en caso contrario ${ }^{26}$.

La proporción de médicos que afirman prescribir AE es inferior a la de otros estudios ${ }^{27,28}$. Creemos que esto puede explicarse por dos motivos: las características sociodemográficas de nuestra población (envejecida y por tanto con una demanda relativamente escasa de AE), con la consiguiente falta de experiencia y/o conocimientos de nuestros profesionales, y la mayor frecuencia de problemas de conciencia (el porcentaje de profesionales que lo consideran un método abortivo en nuestro estudio es elevado y es obvio que esta creencia influye en el hecho de prescribirla o no $)^{1,23,24}$. La no repuesta a la demanda puede tener consecuencias molestas e importantes para la usuaria, al tener que consultar en varios lugares y retrasar el inicio del tratamiento (cuando la máxima efectividad se logra en las primeras 24 horas $^{6,38,42}$ ), disminuyendo su confianza en un Sistema de Salud que no responde a sus necesidades y aumentando la demanda de otros niveles asistenciales.

Es importante destacar el cambio de actitud de los prescriptores habituales cuando la demandante es una menor de edad: la prescripción se reduce al $26,8 \%$ y la mayoría opta por recomendarle volver con un familiar mayor de edad. Creemos que esto se debe al temor a posibles problemas legales. Aunque este debate probablemente persista largo tiempo, en opinión de algunos expertos ${ }^{43}$ y atenién-

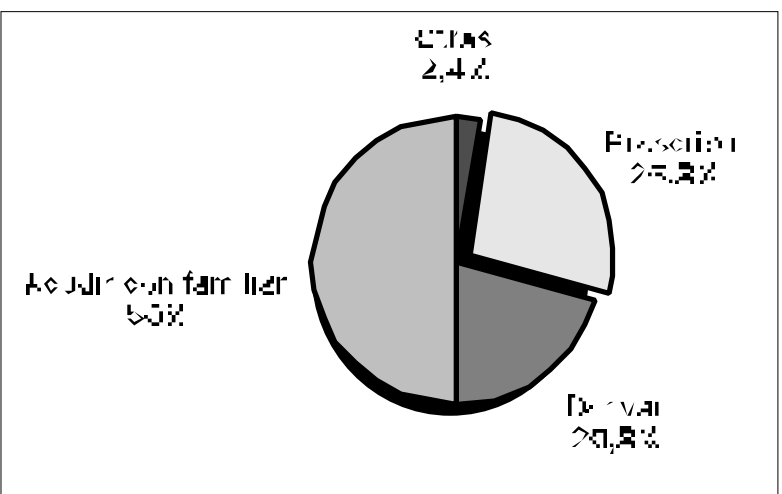

Figura 5

Actitud de los prescriptores habituales de AE si la demanda la hace una mujer menor de edad. 
donos al artículo 10 de la Constitución Española y al artículo 162 del Código Civil -que establece una excepción al régimen de representación y autorización paterna de los menores de edad en los actos relativos a los derechos de la personalidad u otros que el hijo pueda realizar por sí mismo de acuerdo a su madurez-, no habría problemas legales en la prescripción de AE a una menor.

Hay dos aspectos en los que se constata la falta de homogeneidad entre los facultativos a la hora de resolver esta demanda: la prescripción profiláctica de antieméticos y la necesidad de realizar un test de embarazo previo. Desgraciadamente, esto puede deberse a la falta de pautas de actuación claras al respecto. La profilaxis sistemática de las náuseas y los vómitos es recomendada por algunos exper$\operatorname{tos}^{15,16,33,44}$ y desaconsejada por otros ${ }^{4,26}$. Otro tanto sucede con la conveniencia de realizar ${ }^{23} \mathrm{o} \mathrm{no}^{4,37,42}$ test de embarazo previo.

La administración profiláctica de un antiemético ha demostrado reducir la incidencia de náuseas y vómitos en algunas mujeres, mientras que dados después de las náuseas o los vómitos son menos eficaces $^{4,45}$. Sin embargo, como es imposible predecir qué mujeres se beneficiarán y resulta costoso administrar antieméticos a todas las usuarias de AE, la OMS no recomienda el uso profiláctico rutinario de antieméticos ${ }^{1}$. Probablemente sean necesarios estudios que aclaren el tema; hasta entonces, la actitud más razonable, a nuestro juicio, sería darlo a las mujeres que han experimentado previamente náuseas o vómitos al tomar anticonceptivos hormonales o AE.

Respecto a la conveniencia o no de descartar el embarazo mediante un test rápido, los que abogan por su realización se basan en la contraindicación de la AE hormonal en el embarazo; no obstante, dicha contraindicación se debe, no a los posibles efectos adversos en el curso de la gestación, sino a la ineficacia del método en esta situación ${ }^{37}$, por lo que la práctica del test es cuestionable. No existen muchos datos sobre la influencia de la AE en el embarazo, pero la falta de evidencia de teratogenicidad de los anticonceptivos orales y el tiempo en el que se administra (antes de que comience la organogénesis) pueden ser datos tranquilizadores ${ }^{46,47}$. En nuestra opinión, puede ser una actitud prudente la realización del test cuando existen dudas sobre su estado, dado que se trata de una prueba sencilla y poco costosa.

No hemos medido directamente la demanda de AE pero, extrapolándola de los casos demandados a los encuestados en el último año, parece importante (al menos 1.187 demandas/año en un área con 81.000 mujeres entre 15 y 45 años) y acorde con el aumento de solicitudes de AE observado en los últimos años ${ }^{20,48,49}$. Un pequeño porcentaje de nuestros profesionales considera que esta demanda no debe ser asumida por Atención Primaria, sino por el nivel especializado. En nuestra opinión, la AE no requiere conocimientos o habilidades que no estén al alcance del médico de familia y somos el nivel asistencial más accesible y ágil, por lo que, no nos cabe ninguna duda de que debemos incluir la $\mathrm{AE}$ como un servicio más a nuestra población ${ }^{28,49}$. Además, el contacto con estas mujeres puede ser una oportunidad para realizar educación sanitaria sobre sexualidad y anticoncepción ${ }^{50}$.

Como conclusión, existen algunas lagunas de conocimiento sobre la AE en los profesionales de nuestra área, que deben ser cubiertas si queremos atender correctamente a una demanda creciente e importante. Los nuevos métodos de AE (levonorgestrel y tal vez mifepristona en un futuro cercano), muy eficaces y seguros ${ }^{51,52}$, deben ser perfectamente conocidos por los médicos de Atención Primaria. También es necesario construir guías de actuación claras al respecto para unificar la actuación ante esta demanda.

Aunque la AE no debe emplearse como un método anticonceptivo habitual, es una opción que permite subsanar algunos de los fallos que pueden cometerse en la utilización de los mismos y evitar así embarazos no deseados.

\section{CORRESPONDENCIA:}

Francisco López de Castro

Unidad Docente de Medicina de Familia y Comunitaria C/ Barcelona, 2

45005 Toledo

Tel.: 925259908

E-mail: flopez@gapto08.insalud.es 


\section{Bibliografía}

1. Organización Mundial de la Salud. Anticoncepción de emergencia. Guía para la prestación de servicios. Ginebra: OMS; 1999.

2. Llácer A. Mejorar la salud de las mujeres. En: Álvarez Dardet C, Peiró S (eds). La Salud Pública ante los desafíos del nuevo siglo. Informe SESPAS 2000. Granada: Escuela Andaluza de Salud Pública, 2000; 133-49.

3. Brandrup-Lucanow A. Entre nous. Publicación del Servicio de las Mujeres y la Salud Reproductiva de la Oficina Regional Europea de la OMS 1998; 39: 2.

4. Consortium for Emergency Contraception. Píldoras anticonceptivas de emergencia. Guías Médicas y de Entrega de Servicios [en linea]. <http://www.path.org/cec/html/es_guiasmedicas.htm > [Consulta: 8 mar. 2001].

5. Association of Reproductive Health Professionals. Reproductive Health [en linea]: An Update. Emergency Contraception Update. Clinical Proceedinngs (electronic edition) August 2000. <http://www.arhp.org/updatefreme.html> [Consulta: 21 mar. 2001].

6. Gy. Bártfai. Emergency contraception in clinical practice: global perspectives. International Journal of Gynecology \& Obstetrics 2000; 70: 49-58.

7. Yuzpe AA, Lancee WJ. Ethinylestradiol and dl-norgestrel as a postcoital contraceptive. Fertil Steril 1977; 28: 932-6.

8. Ramirez Hidalgo A. Intercepción postcoital. Formación Médica Continuada 2000; 7: 187-94.

9. Piaggio G, von Hertzen H, Grimes DA, Van Look PFA. Timing of emergency contraception with levonorgestrel deYuzpe regimen. Lancet 1999; 353: 721.

10. Rodrigues I, Grou F, Joly J. Effectiveness of emergency contraceptive pills between 72 and 120 hours after unprotected sexual intercourse. Am J Obstet Gynecol 2001; 184 (4): 531-7.

11. Cheng L, Gülmezoglu AM, Ezcurra E, Van Look PFA. Interventions for emergency contraception. Oxford: The Cochrane Library, Issue 4. Update Software 2000.

12. Gynetics. Inc. Pescribing information [en linea]. The PREVEN Emergency Contraceptive Kit consisting of Emergency Contraceptive Pills And Pregnancy Test. Somerville, 1998. <http://www.preven.com/product/02-06.html> [Consulta: 2 mar. 2001].

13. Trussell J, Ellertson C, Rodríguez G. The Yuzpe regimen of emergency contraception: how long after the morning after? Obstet Gynecol 1996; 88 (1): 150-4.

14. Wellbery C. Emergency Contraception. Arch Fam Med 2000; 9: 642-6.

15. Adolescent Medicine Committee, Canadian Paediatric Society. Emergency contraception. Paediatrics \& Child Health 1998; 3 (5): 359-61.

16. The Canadian Consensus Conference on Contraception. Journal SOGC 1998; 20 (5-8): 1-71.

17. Ho PC. Emergency contraception: methods and efficacy. Curr Opin Obstet Gynecol 2000; 12 (3): 175-9.

18. Trussell J, Rodríguez G, Ellertson C. Updated estimates of the effectiveness of the Yuzpe regimen of emergency contraception. Contraception 1999; 59 (3): 147-51.

19. Hernandez Velasco I. En busca de la píldora del día después. El Mundo, 20 abril 1997. También disponible en <www.elmundo.es/1997/04/20/sociedad/ 20N0042.html> [Consulta: 4 jun. 2001].

20. Salom A. La píldora postcoital, a la venta en febrero. 7DM, 1 diciembre 2000; 42 (col 2-3).

21. Grimes DA. Updates in contraception from the XVI World Congress of the Federation of Gynecologyc and Obstetrics. Medscape Womens Health 2000; 5(5): E3.

22. Consellería de Sanitat de la Generallitat Valenciana. Anticoncepción postcoital [en línea]. Boletin Red Centinela no11 1999. Editado el 20 de enero de 2000. <http://dgsp. san.gva.es/SSCC/Epidemiologia/CENTINELAS/RCS99/B OLCEN11. htm> [Consulta: 25 jul. 2000].

23. Golden NH, Seigel WN, Fischer M, Schneider M, Quijano E, Suss A, et al. Emergency Contraception: Pediatrician's knowledge, attitudes and opinions. Pediatrics 2001; 107 (2): 287-92.

24. Sueiro E, Perdiz C, Gayoso P, Doval JL. Uso y efectos de la anticoncepción postcoital. Aten Primaria 1997; 20 (6): 305-10.

25. Burton R, Savage W. Knowledge and use of postcoital contraception: a survey among health professionals in Tower Hamlets. Br J Gen Pract 1990; 40 (337): 326-30.

26. Glasier A. Emergency Postcoital Contraception. N Engl J Med 1997; 337 (15) : 1058-65.

27. Wisberg E, Fraser I, Carrick S, Wilde F. Emergency Contraception: General practitioner knowledge, attitudes and practices in New South Wales. The Medical Journal of Australia $1995 ; 162$ : 136-8.

28. Carlón López R, Gracía Houghton RF, Gónzalez Varela A, Martínez Suárez MM, Suárez Gil P. Intercepción postcoital hormonal: estudio sobre actitudes y prescripción de los médicos de atención primaria. Aten Primaria 2000; 26(9): 595-9.

29. Cheng D. The intrauterine device: still misunderstood after all these years. South Med J 2000; 93 (9): 859-64.

30. Reuter S. Barriers to the use of IUDs as emergency contraception. Br J Fam Plann 1999; 25 (2): 61-8.

31. Trussell J, Ellertson C. Efficacy of emergency contraception. Fert Control Rev 1995; 4: 8-11.

32. López de Castro F, Campos Campos N, Castillo Portales S, Rodríguez Alcalá FJ. Después del levonorgestrel, ¿será la mifepristona (RU486) la siguiente "píldora del día después"? Aten Primaria 2001; 2 (5): 368.

33. National Guideline Clearinghouse. Emergency oral contraception [en linea]. <http://www.guideline.gov/FRAMESETS/guideline_fs.asp?view=full_summary\&guideline $=000422 \&$ sSearch_string=emergency+oral+contraception> [Consulta: 12 mar. 2001].

34. ACOG practice patterns. Emergency oral contraception. International Journal of Gynecology \& Obstetrics 1997; 56: 203-10.

35. Cooling H. Emergency Contraception. Update 1999; 58 (10): 976-82

36. Vasilakis C, Jick S, Jick H. The Risk of Venous Thromboembolism in Users of Postcoital Contraceptive Pills. Contraception 1999; 59: 79-83.

37. Glasier A. Safety of Emergency contraception. Jamwa 1998; 53 (5): 219-21.

38. Von Hertzen E, Van Look FA. Randomised controlled trial of levonorgestrel versus the Yuzpe regimen of combined oral contraceptives for emergency contraception. Lancet 1998; 352: 428-33.

39. Webb A, Russell J, Elstein M. Comparison of Yuzpe regimen, danazol, and mifepristone (RU-486) in oral postcoital contraception. BMJ 1992; 305: 927-31.

40. Apgar BS, Greenberg G. Using progestins in clinical practice. Am Fam Physician 2000 PCT 15; 62 (8): 1839-46.

41. Apgar BS, Greenberg G. Using progestins in clinical practice. Am Fam Physician 2000 PCT 15; 62 (8): 1849-50.

42. Davis V, Dunn S. Emergency Postcoital Contraception. Journal SOGC 2000; 92: 1-5.

43. García Morillo J. Aspectos legales de la planificación familiar en la adolescencia (ponencia). Actas del II Congreso Estatal de la Federación de Planificación Familiar de España; 1987 abril. Valladolid: Federación de Planificación Familiar de España, 1987.

44. Gold MA. Emergency contraception. Adv Pediatr 2000; 47: 309-34. 
45. Raymond EG, Creinin MD, Barnhart KT, Lovvorn AE, Rountree RV, Trussell J. Meclizine for prevention of nausea associated with use of emergency contraceptive pills: a randomized trial. Obstet Gynecol 2000; 95 (2): 271-7.

46. Bracken MB. Oral contraceptions and congenital malformations in offspring: A review and meta-analysis of the prospective studies. Obstet Gynecol 1990; 76 (3 Pt 2): 552-7.

47. Kubba AA. Hormonal postcoital contraception. Eur J Contracept Reprod Health Care 1997; 2 (2): 101-4.

48. Ineichen B, Logie J, Rowlands S, Lawrenson R. Patterns of prescription of PC4 by general practitioners in England and
Wales. Eur J Contracept Reprod Health Care 2000; 5(4): 241-7.

49. Ramírez Puerta DN, Zarco Rodríguez JV. Doctor, ¿puede mandarme la píldora? (editorial). Semergen 2001; 27: 3-4.

50. Klima CS. Emergency contraception for midwifery practice. J Nurse Midwifery 1998; 43 (3): 182-9.

51. Spitz IM, Van Look PF, Coelingh Bennink HJ. The use of progesterone antagonists and progesterone receptor modulators in contraception. Steroids 2000; 65 (10-11): 817-23.

52. Guillebaud J. Time for emergency contraception with levonorgestrel alone. The Lancet 1998; 352: 416-7. 